\title{
Spontaneous CSF Collection in the Cervical Spine may Cause Neurological Deficit and Intra-cranial Hypotension
}

\author{
Rasheed Zakaria $^{1, *}$, Martin Wilby ${ }^{1}$, Nicholas A Fletcher ${ }^{2}$ \\ ${ }^{1}$ Department of Neurosurgery, The Walton Centre NHS Foundation Trust, Liverpool, UK \\ ${ }^{2}$ Department of Neurology, The Walton Centre NHS Foundation Trust, Liverpool, UK
}

\begin{abstract}
Objective: a case is described of a spontaneously occurring cerebrospinal fluid collection in the ventral cervical spine which caused radiculopathy and spontaneous intracranial hypotension.

Case: a sixty eight year old Caucasian man presented with a 2 year history of proximal upper limb weakness with a history of trivial cervical trauma many years previously.

Methods: the patient was investigated with blood tests for causes of peripheral neuropathy, nerve conduction and electromyography studies, lumbar puncture and MRI of the brain and spine with contrast.

Results: a cerebrospinal fluid collection was identified in the ventral cervical spinal cord causing mass effect associated with cord atrophy and there were signs of spontaneous intracranial hypotension on the MRI brain including subdural cerebrospinal fluid collections, meningeal enhancement and slumped posterior fossa.

Conclusions: this is the first description of a spontaneous spinal fluid collection causing direct compression and cord signal change, manifest as a motor deficit, with intracranial signs of spontaneous hypotension. Spinal imaging is recommended in cases of spontaneous intracranial hypotension and cerebrospinal fluid collections in the spine may rarely be a cause of radiculopathy in such cases.
\end{abstract}

Keywords: Cerebrospinal fluid, radiculopathy, spontaneous intracranial hypotension.

\section{CASE DESCRIPTION}

A 68 year old right hand dominant, Caucasian man was referred to the neurology service with a 2 year history of arm weakness. The patient had noticed increasing difficulty lifting items of high shelves, carrying luggage or shopping. There was no associated pain or loss of sensation in the arms. Dexterity was unaffected. His walking, lower limbs and bladder/bowel control were unaffected. He had no other neurological symptoms except mild postural headache. There was no history of previous spinal surgery or intervention. He was taking no regular medications. At examination, cranial nerves were intact, wasting was seen at the deltoids with weakness in myotomes C5 and C6, MRC grade 3/5. Biceps jerk was reduced bilaterally. Reflexes were diminished in the lower limbs but sensation, power and gait were normal.

The patient underwent investigations for myositis and peripheral neuropathy with blood tests including electrolytes, renal function, full blood count, calcium, liver enzymes, erythrocyte sedimentation rate, vitamin B12, fasting glucose,

*Address correspondence to this author at the Department of Neurosurgery, The Walton Centre NHS Foundation Trust, Lower Lane, Fazakerley, Liverpool, L9 7LJ; Tel: 0151525 3611; Fax: 0151529 5500;

E-mail: rzakaria@nhs.net serum electrophoresis and auto-antibodies. All of these were normal. MRI of the brain, shown in Fig. (1a) demonstrated enhancement of the meninges intracranially. Also seen on other slices were bilateral subdural CSF collections and sagging of the posterior fossa structures. A lumbar puncture demonstrated no abnormalities on cell count, cytology or microbiology and the opening pressure was in the normal range. Electromyography and nerve conduction studies showed bilateral severe C5 and C6 radiculopathies. MRI whole spine was subsequently ordered and Fig. (1b) demonstrated a cervical cord fluid collection anteriorly at C2-C5 with signal isointense to CSF on T1, T2 and T2 FLAIR sequences, associated with signal change and atrophy of the cord at those levels. There was no enhancement with gadolinium administration. CT myelogram confirmed the collection to be CSF with pressure on the C5 and C6 roots, but no leaking point was identified. Blood patch was therefore not undertaken. The patient's neurological deficit remained stable and he did not want any further intervention, undergoing physical therapy only. Subsequent MRI scan at 6 months has shown no change in the size of the fluid collection and no change in the intracranial appearances.

\section{DISCUSSION}

Spontaneous intracranial hypotension $(\mathrm{SIH})$ is usually characterised by orthostatic headache and intracranial imag- 

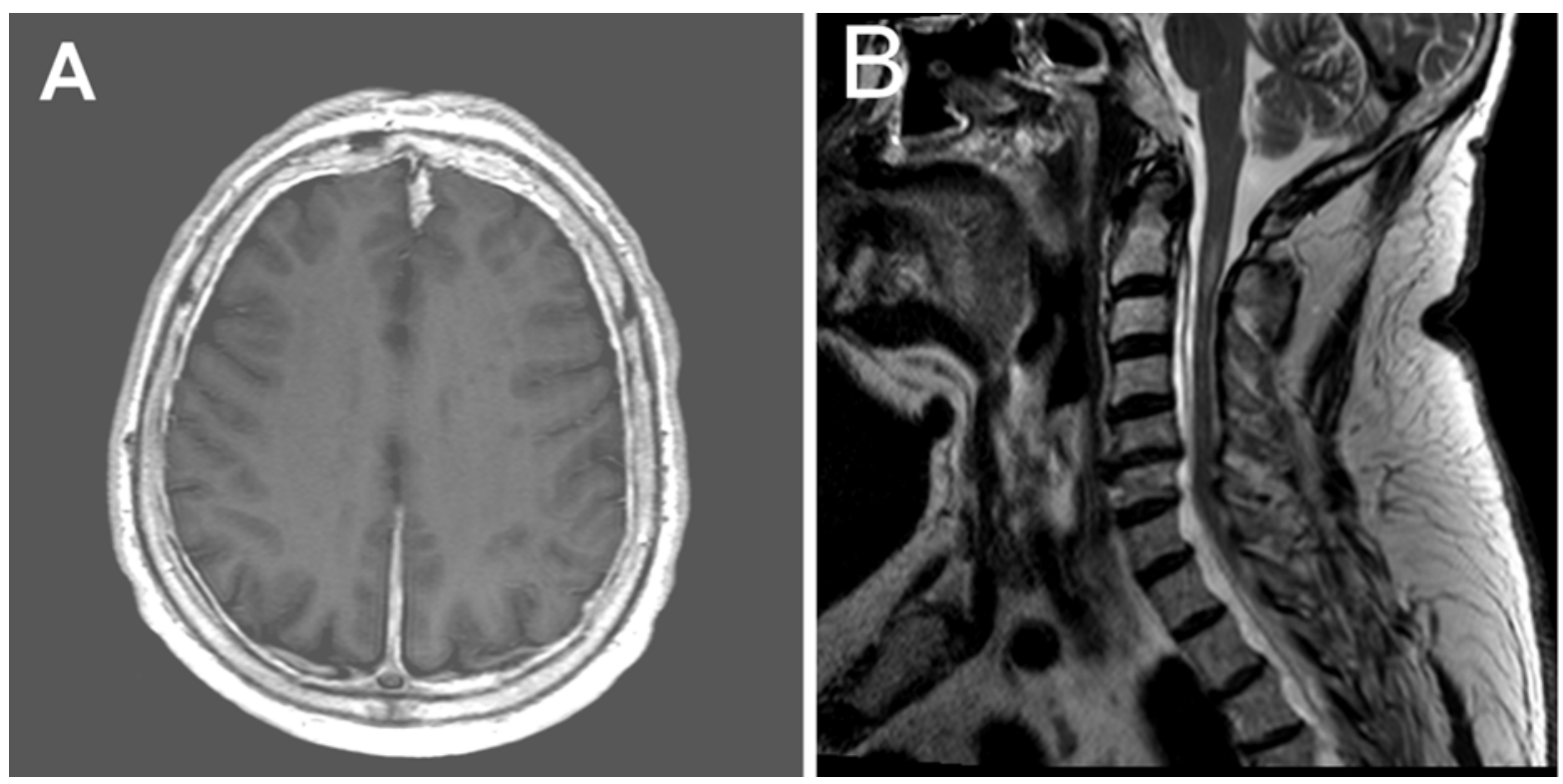

Fig. (1). A. Axial T1 weighted MRI brain with gadolinium showing strong enhancement of the meninges. B. Sagital T2 weighted MRI of the craniocervical junction showing crowded posterior fossa with steeply sloping tentorium, cord atrophy from approximately C2 to C5 level with intrinsic signal change and a fluid collection likely to be a subdural CSF collection, anterior to the cord from C2 to C5.

ing findings of subdural hygroma, meningeal enhancement and slump of the hindbrain. All of these features were seen in this case but in addition there were spinal imaging abnormalities and in particular a ventral cervical spine CSF collection which seems to have caused bilateral radiculopathy. This collection may have been subdural or extradural though the imaging suggests the former and the signal characteristics are isointense to CSF on both T1 and T2. Radiologically the differential for such a collection would include epidural haematoma, abscess, arachnoid cyst and neuroenteric cyst. CSF was taken to rule out malignant meningitis and gadolinium enhanced MRI of the spine and inflammatory markers did not suggest a chronic infective collection.

Although subdural spinal CSF collections have been described, this is the first time one has been reported in association with florid changes in the cord with atrophy and signal abnormality at the relevant level. Two recent reports $[1,2]$ have suggested patients may present in a delayed fashion with symptoms of low CSF pressure and spinal fluid collections causing mass effect but there has been no direct evidence of cord compromise on imaging. There is already a recognition that spinal imaging may reveal abnormalities in patients with SIH and one large series showed 16 of 18 patients with SIH had spinal CSF fluid collections most commonly anterior to the cord in the cervical region, though none of these were compressive and no patients had attributable neurological deficits as a result [3]. Similarly in a prospective cohort, 6 of 9 patients with SIH were seen to have spinal hygroma of which only half resolved with treatment. Again, there was no focal cord compression clinically or radiologically [4]. A further series of 3 cases described one patient with possible cord compression and Lhermitte's sign but again there was no signal change in the cord and the fluid collection was extensive [5].

The origin of spinal CSF collections in SIH is controversial. They may simply be manifestations of direct CSF leak- age either from a single point or diffusely through a damaged arachnoid and dural membrane. It can be suggested that these collections are a transudate with high protein content and in association with venous plexus engorgement as CSF is drawn out into the epidrual or subdural space. It is intriguing that here the cord appeared atrophic and this raises the possibility of chronic pressure either from the collection anteriorly or in a dynamic fashion with flexion of the neck, as is said to occur in Hirayama's disease [2].

\section{CONCLUSION}

This is the first description of a spontaneous spinal fluid collection causing direct compression and cord signal change, manifest as a motor deficit with intracranial signs of spontaneous intracranial hypotension. It raises the intriguing possibility of patients with SIH presenting with neurological deficits arising from spinal fluid collections in the absence of any headache and in line with recent reports suggests spinal MRI is a useful investigation in this condition.

\section{CONFLICT OF INTEREST}

No funding was received for this work and the authors have no conflict of interest to declare.

\section{ACKNOWLEDGEMENT}

None Declared

\section{REFERENCES}

[1] Mihaylova T, Biondo A, Zak I, Lewis RA. Anterior horn cell loss from subdural hygroma: a consequence of spontaneous spinal fluid leak. J Neurol sci 201; 305(1-2):156-9.

[2] Deluca GC, Boes CJ, Krueger BR, Mokri B, Kumar N. Ventral intraspinal fluid-filled collection secondary to CSF leak presenting as bibrachial amyotrophy. Neurology 2011; 76(16):1439-40. 
[3] Watanabe A, Horikoshi T, Uchida M, Koizumi H, Yagishita T, Kinouchi H. Diagnostic value of spinal MR imaging in spontaneous intracranial hypotension syndrome. Am J Neuroradiol 2009; 30(1): 147-51.

Received: February 04, 2012 Revised: March 28, 2012
Yousry I, Förderreuther S, Moriggl B, et al. Cervical MR imaging in postural headache: MR signs and pathophysiological implications. Am J Neuroradiol 2001; 22(7):1239-50.

[5] Rabin BM, Roychowdhury S, Meyer JR, et al. Spontaneous intracranial hypotension: spinal MR findings. Am J Neuroradiol 1998; 19(6):1034-9.

(C) Zakaria et al.; Licensee Bentham Open.

This is an open access article licensed under the terms of the Creative Commons Attribution Non-Commercial License (http://creativecommons.org/licenses/by-nc/3.0/) which permits unrestricted, non-commercial use, distribution and reproduction in any medium, provided the work is properly cited. 